\title{
Immune-modulating efficacy of a polyphenol-rich beverage on symptoms associated with the common cold: a double-blind, randomised, placebo-controlled, multi-centric clinical study
}

\author{
Katrin Schütz ${ }^{1}$, Matthias Saß ${ }^{1}$, Axel de With ${ }^{1}$, Hans-Joachim Graubaum ${ }^{2}$ and Jörg Grünwald ${ }^{2}$ \\ ${ }^{1}$ Rudolf Wild GmbH \& Co. KG, Rudolf-Wild-Straße 107-115, 69214 Eppelheim, Germany \\ ${ }^{2}$ analyze \& realize ag, Waldseeweg 6, 13467 Berlin, Germany
}

(Received 26 January 2010 - Revised 13 April 2010 - Accepted 19 April 2010 - First published online 21 May 2010)

In the present study, the immune-modulating efficacy of a polyphenol-rich beverage on symptoms associated with the common cold was evaluated. For this purpose, ninety-eight patients reporting common cold symptoms that began no longer than $24 \mathrm{~h}$ before the study intervention were randomly assigned to consume either the test beverage or placebo twice per $\mathrm{d}$ for $10 \mathrm{~d}$. The severity of the disease was expressed as the total score of the five cold symptoms 'general feeling of sickness', 'headache and/or joint aches', 'sore throat and/or difficulty swallowing', 'hoarseness and/or cough' and 'stuffy nose/sniffle'. Consequently, the decrease from $10 \cdot 2$ (SD 3.1) points at the beginning to $2 \cdot 1$ (SD $2 \cdot 7$ ) points by the end of the study in the verum group demonstrated a clear improvement, whereas in the placebo group only a reduction from 10.5 (SD 3.0 ) to 6.3 (SD 3.8 ) points could be observed. The mean difference between the groups (primary efficacy criterion) of 3.9 points was highly significant $(P<0.01)$. At the end of the study there were highly significantly $(P<0.01)$ more patients in the verum group complaint free than in the placebo group (secondary efficacy criterion). In addition to these self-reported values, several local findings of the physical examination were also significantly improved in the verum group.

Immune-modulating efficacy: Polyphenols: Beverages: Common cold: Soft drinks

Acute upper respiratory tract viral infection, also referred to as the common cold, is the most frequent infectious disease in human beings. The majority of adults are affected two to four times, and children even six to eight times per year, resulting in economic losses due to absence at work or school. The mean duration of a common cold is 7 to $10 \mathrm{~d}$ whereas the peak level of symptom severity is reached 2 to $3 \mathrm{~d}$ after incubation ${ }^{(1)}$. Symptoms often start with headache, sneezing, chilliness and sore throat followed by nasal discharge, nasal obstruction, cough and general feeling of sickness $^{(2)}$. Due to the diversity of the viral causes and the fact that the common cold is usually self-limiting, treatment has mainly focused on reducing the duration and severity of symptoms. Besides conventional treatment with overthe-counter analgesics, nasal and/or oral decongestants and antipyretics, self-medication with botanicals is popular ${ }^{(3,4)}$ Particularly, Echinacea and elder are used based on long experience in folk medicine as well as scientific investigations including human studies ${ }^{(5-9)}$. The health-promoting potential is mainly attributed to the immunostimulative, antiviral, anti-inflammatory and antioxidative activity of low- and high-molecular-weight compounds such as flavonoids and polysaccharides $^{(10-12)}$.

Flavonoids, particularly catechins, are also the active principle of green tea and green tea extracts ${ }^{(13)}$. In several in vitro studies the immunomodulatory effects of green tea catechins through immunostimulatory ${ }^{(14,15)}$ as well as antiinflammatory $^{(16)}$, antiviral ${ }^{(17,18)}$ and antibacterial ${ }^{(19)}$ activities were proven. Furthermore, these findings were affirmed in human studies. In a prospective clinical study, gargling with tea catechin extract significantly reduced the incidence of influenza infection compared with gargling without tea catechin extract in elderly subjects ${ }^{(20)}$. Stimulation of immune cell function after consumption of a high-polyphenol juice containing green tea catechins was confirmed by increased lymphocyte proliferative responsiveness, IL-2 secretion by activated lymphocytes as well as enhanced lytic activity of natural killer cells in a further randomised study involving twenty-seven healthy men. Additionally, reduced oxidative DNA damage in lymphocytes due to antioxidative activities of phenolic compounds was observed ${ }^{(21)}$. The capability to increase the antioxidative capacity in healthy subjects is probably the most investigated pharmacological effect of polyphenols from grapes $^{(22-26)}$. Moreover, as for green tea polyphenols, several in vitro findings and animal experiments support the immunomodulatory effects of phenolic compounds from grapes. Intragastrically applied grape seed proanthocyanidins significantly inhibited tumour growth and affected immune functions through increased natural killer cell cytotoxicity, lymphocyte proliferation, $\mathrm{CD}^{+}: \mathrm{CD}^{+}$ratio, IL- 2 and interferon- $\gamma(\mathrm{IFN}-\gamma)$ 
production in tumour-bearing mice ${ }^{(27)}$. Elevated IFN- $\gamma$ production together with enhanced IFN- $\gamma$ gene expression and increased regulated number of IFN- $\gamma$-positive cells have already been observed in an earlier study investigating the influence of grape seed extract on peripheral blood mononuclear cells from healthy donors ${ }^{(28)}$. In addition to such direct effects on the immune system, antiviral as well as antibacterial properties have been described for various grape products and grape seed extracts ${ }^{(29-31)}$.

Immune-stimulating activity is not only attributed to polyphenols but also to polysaccharides. Particularly, the property to trigger both the innate and adaptive immune system of lentinan, a $\beta-1,6 ; \beta-1,3$-glucan from shiitake mushrooms, is well documented by numerous in vitro tests. The mechanism is postulated to involve $\beta$-glucan binding to lymphocyte surfaces or serum-specific proteins, which activates macrophages, T-helper cells, natural killer cells, and other effector cells. Consequently, the production of antibodies as well as interleukins (IL-1, IL-2) and IFN- $\gamma$ is increased ${ }^{(32)}$. This stimulation of the immune system by fungal glucans is accounted to be the active principle in treating cancer and infectious diseases ${ }^{(33)}$.

In view of these findings, a polyphenol-rich beverage containing green tea (Camellia sinensis), grape seed and grape skin (Vitis vinifera L.) as well as shiitake mushroom (Lentinus edodes) extract was created and its immune-stimulating activity was investigated in patients suffering from the common cold. Since no single immune marker that accurately reflects an individual's resistance to infection exists, the recommendation of the PASSCLAIM publication on immunity was followed and the severity and duration of the disease were assessed ${ }^{(34,35)}$.

\section{Materials and methods}

\section{Subjects}

Volunteers with a common cold were recruited via advertisements, in practices of the clinical investigators, and from pharmacies that had previously received information material from the contract research organisation. Included were 100 patients aged between 20 and 65 years scoring in total at least 5 points on the severity of five cold symptoms (maximum 15 points; see below) and having present at least one cold-related local finding (throat). Exclusion criteria consisted of the following: active allergic rhinitis or asthma, fever (body temperature $>39^{\circ} \mathrm{C}$ ), clinically relevant laboratory value deviations indicating severe organ or system disease, bacterial tonsillitis, pyorrhoea on the back of the throat, cancer or AIDS (HIV positive), pregnancy or nursing, alcohol, medication or drug dependence, participation in a clinical study within the previous $30 \mathrm{~d}$, intake of immune suppressants, immune stimulants, analgesics/anti-rheumatics, anti-inflammatories, antitussives, expectorants, influenza remedies, mouth and throat therapeutics as well as inability to comply due to language difficulties.

The patients gave their informed written consent to participate in the study. The study was approved by the ethical committee of the University Hospital Charite of the Humboldt-University Berlin, Germany, and performed according to the principles of the World Medical Association (Declaration of Helsinki/Hong Kong/Somerset), the German Drug Law (Arzneimittelgesetz; AMG) and the European Union recommendations (CPMP/ICH/135/95).

\section{Study design}

The study was performed between February and August 2004, to coincide with the normal season for the common cold. Investigation was conducted as a randomised, prospective, placebo-controlled, multi-centric clinical, double-blind study. A randomisation diagram with a block size of four, and in a parallel-group design with two independent treatment groups was created using EDGAR (Experimental Design Generator and Randomiser; James KM Brown, John Innes Centre, Norwich, UK; http://www.edgarweb.org.uk). Patients were instructed to consume $2 \times 250 \mathrm{ml}$ of the verum or the placebo beverage daily. The trial was accomplished within $10 \mathrm{~d}$. Examinations through clinical investigators were scheduled immediately before the start of treatment (first examination, baseline), after 3 to $6 \mathrm{~d}$ (second examination) and finally after 7 to $10 \mathrm{~d}$ (third examination).

\section{Compliance control}

Compliance control was performed on the basis of the empty packaging and unused investigational product, which was documented for each patient. Furthermore, a patient was rated as compliant when he/she carefully filled out the patient diary and followed the assigned examination appointments.

\section{Efficacy evaluation}

The primary efficacy criterion was defined as the decrease of the total score of the five cold symptoms (headache and/or joint pains, sore throat and/or difficulty swallowing, hoarseness and/or cough, nasal congestion/sniffle) in comparison with placebo, displaying an improvement of the disease. It was assessed by the physician by questioning.

'Complaint free by the end of the study' was specified as the secondary efficacy criterion. For this criterion, the appraisal of the physician and the patient diary were evaluated.

During the three examinations, the total score of the five common cold symptoms was determined by summation of the single scores. The severity of the symptoms was rated on a scale from 0 to 3 points $(0=$ not present; $1=$ mild; $2=$ moderate; $3=$ strong), giving a theoretical sum score range from 0 to 15 . Furthermore, the severity of the concurrent variables, such as the local findings 'reddening of the Waldeyer's ring or tonsils', 'granulation and/or myxorrhoea on the pharyngeal wall' and 'herpes labialis' was categorised as 'not present' ( 0 points), 'mild' ( 1 point) or 'strong' ( 2 points) by the physician. In addition to the medical examinations, the patients were required to record daily, mornings and evenings, the changes of the above-mentioned cold symptoms as well as the concurrent variables 'disturbance of daily activities', 'sleep disorders', 'additional concurrent medication' and 'the number of tissues used' for the duration of the study or until an early complaints-free condition could be documented.

\section{Safety evaluation}

The safety of the investigational product was assessed by clinical data (weighing, measurement of pulse, blood pressure, and body temperature), safety parameters (Hb, packed cell volume, erythrocytes, blood platelets, alanine 
aminotransferase, aspartate aminotransferase, $\gamma$-glutamyltransferase, total bilirubin, creatinine, uric acid, and total protein), the occurrence of adverse events, as well as the global evaluations of the patients and clinical investigators.

\section{Test material}

The placebo drink was a red-coloured soft drink containing water, sugar, citric acid and flavour. Compared with the placebo drink, the verum drink was standardised with respect to the content of polyphenols $(1400 \mathrm{mg} / \mathrm{l}$ measured according to Folin-Ciocalteu and expressed as gallic acid equivalents (GAE)). The verum drink contained green tea extract $(3 \mathrm{~g} / \mathrm{l})$, grape peel extract $(12 \mathrm{~g} / \mathrm{l})$, grape seed extract $(0.5 \mathrm{~g} / \mathrm{l})$, shiitake mushroom extract $(0.05 \mathrm{~g} / \mathrm{l})$ as well as vitamin $\mathrm{C}(0.3 \mathrm{~g} / \mathrm{l})$. The verum and placebo drinks were identical in visual appearance, flavour, as well as brix and titre values, and were packaged in $250 \mathrm{ml}$ cans. Product identity was not revealed to the study investigators and patients until after completion of the study. Sample size was adapted to the normal serving size of beverages.

\section{Analytical parameters}

Antioxidative activity in both verum and placebo was determined as Trolox-equivalent antioxidant capacity (TEAC) ${ }^{(36)}$, ferric-reducing ability of plasma (FRAP) ${ }^{(37)}$ and photochemiluminescence ${ }^{(38)}$. Furthermore, individual polyphenols were quantified by HPLC, and total polyphenols, expressed as GAE, were measured according to the Folin-Ciocalteu method in both the verum and placebo as well as the raw materials green tea, grape skin and seed extracts ${ }^{(39)}$. Ascorbic acid analysis was carried out as previously reported ${ }^{(40)}$.

\section{Statistical analysis}

All analyses were carried out in concordance with current good clinical practice guidelines and were given for all variables. Evaluation was performed using the $t$ test and $\chi^{2}$ test according to the intention-to-treat principle. For each comparison, $P<0.05$ was considered to be statistically significant.

\section{Results}

\section{Study group characteristics}

Since no case report forms were received for two participants, the patient number was reduced to ninety-eight patients. Therefore, the verum and placebo groups comprised fortynine patients each. For both groups there was a uniform baseline in reference to age, height, body weight and temperature (Table 1). Moreover, two other patients, both in the placebo group, prematurely terminated the study. They did not appear for the second and third examination, and hence remained without final examination. Data from the patient missing only the final examination were included, whereas those from the other patient were not considered for analysis.

\section{Compliance control}

All patients remained in the study for the required duration, returned the empty packaging as well as unused investigational
Table 1. Sociodemographic data of all volunteers, the verum group and placebo group

(Mean values and standard deviations)

\begin{tabular}{|c|c|c|c|c|c|c|}
\hline \multirow[b]{2}{*}{ Parameter } & \multicolumn{2}{|c|}{$\begin{array}{l}\text { All volunteers } \\
\quad(n 98)\end{array}$} & \multicolumn{2}{|c|}{$\begin{array}{l}\text { Verum } \\
(n 49)\end{array}$} & \multicolumn{2}{|c|}{$\begin{array}{l}\text { Placebo } \\
(n 49)\end{array}$} \\
\hline & Mean & SD & Mean & SD & Mean & SD \\
\hline Age (years) & $40 \cdot 8$ & $12 \cdot 9$ & $40 \cdot 8$ & $12 \cdot 8$ & $40 \cdot 8$ & $13 \cdot 1$ \\
\hline Height $(\mathrm{cm})$ & $172 \cdot 9$ & 8.5 & 173.8 & 9.1 & $172 \cdot 0$ & 7.9 \\
\hline Body weight $(\mathrm{kg})$ & $70 \cdot 4$ & $12 \cdot 3$ & 71.5 & 11.6 & 69.4 & $13 \cdot 1$ \\
\hline
\end{tabular}

product completely and filled out the diary carefully. The only exceptions were the two patients who did not appear for the second and third examination, and one further patient, who did not return the patient diary. All three patients were in the placebo group.

\section{Safety evaluation}

No significant changes for any safety parameters and clinical data neither in the verum nor in the placebo group were observed during the course of the study, except for body temperature in the verum group which showed a highly significant reduction $(P=0.003)$ but remained in the normal temperature range. In respect of adverse events, only one patient mentioned 'sour burps' and nausea immediately after taking the placebo product on the first day. The complaints occurred repeatedly for $2 \mathrm{~d}$ but were of mild intensity and did not lead to premature termination of the study. Global safety evaluation revealed 'very good' and 'good' tolerability for the verum and placebo drinks, respectively, as rated by the patients as well as the clinical investigators.

\section{Composition of verum and placebo as well as total phenolic contents of raw material extracts}

Table 2 shows the concentration of individual polyphenols, total phenols, and ascorbic acid content in the verum and placebo products. Furthermore, the results of antioxidative activity measurements are summarised. The total phenol content of green tea, grape skin and grape seed extract was

Table 2. Analytical parameters of placebo and verum beverages

\begin{tabular}{lcr}
\hline Parameter & Placebo & Verum \\
\hline Antioxidative activity & & \\
PCL (mmol Trolox/l) & $0 \cdot 24$ & $9 \cdot 78$ \\
TEAC (mmol Trolox/l) & $0 \cdot 11$ & $7 \cdot 65$ \\
FRAP (mmol Fe+/l) & $1 \cdot 75$ & $51 \cdot 72$ \\
Total phenols (mg/l) & 91 & 1437 \\
Polyphenols (by HPLC) & & \\
Gallic acid (mg/l) & $<1$ & $77 \cdot 7$ \\
Catechin (mg/l) & n.d. & $35 \cdot 7$ \\
EGCG (mg/l) & n.d. & $127 \cdot 2$ \\
EC (mg/l) & n.d. & $54 \cdot 5$ \\
ECG (mg/l) & n.d. & $28 \cdot 5$ \\
Ascorbic acid (mg/l) & n.d. & $122 \cdot 7$ \\
\hline PCL, photochemiluminescence; TEAC, Trolox-equivalent \\
$\quad$ antioxidant capacity; FRAP, ferric-reducing ability of \\
plasma; n.d., not detectable; EGCG, epigallocatechine \\
gallate; EC, epicatechin; ECG, epicatechin gallate.
\end{tabular}


Table 3. Total score of cold symptoms during the course of the study (Mean values and standard deviations)

\begin{tabular}{|c|c|c|c|c|c|c|c|c|c|c|}
\hline \multirow[b]{2}{*}{ Examination } & \multicolumn{3}{|c|}{ Total collective } & \multicolumn{3}{|c|}{ Verum group } & \multicolumn{3}{|c|}{ Placebo group } & \multirow[b]{2}{*}{$P^{*}$} \\
\hline & $n$ & Mean & SD & $n$ & Mean & SD & $n$ & Mean & SD & \\
\hline 1 & 97 & $10 \cdot 4$ & 3.0 & 49 & $10 \cdot 2$ & 3.1 & 48 & 10.5 & 3.0 & 0.630 \\
\hline 2 & 97 & 7.9 & $3 \cdot 3$ & 49 & 6.7 & 2.5 & 48 & 9.2 & 3.6 & 0.001 \\
\hline 3 & 97 & 4.2 & 3.9 & 49 & $2 \cdot 1$ & 2.7 & 48 & $6 \cdot 3$ & 3.8 & 0.001 \\
\hline
\end{tabular}

*Verum group $v$. placebo group $(t$ test).

$164185 \mathrm{GAE} \mathrm{mg} / \mathrm{kg}, 45120 \mathrm{GAE} \mathrm{mg} / \mathrm{kg}$ and $39286 \mathrm{GAE}$ $\mathrm{mg} / \mathrm{kg}$, respectively.

\section{Improvement of cold symptoms (primary efficacy criterion)}

Total score was obtained by summarising the score of the five cold symptoms headache and/or joint pains, sore throat and/or difficulty swallowing, hoarseness and/or cough, and nasal congestion/sniffle, which were evaluated by the clinical investigator during the three examinations. The improvement is reflected in the reduction of the total score between the first and the second as well as the first and the third examination (Table 3), whereby the comparison of the mean group difference in the latter case revealed the primary efficacy criterion. At the beginning of the study, the mean total score was 10 points for both the verum and placebo group, displaying a homogeneous baseline situation. Already at the second examination a highly significant difference $(P<0.001)$ between the mean total score of both groups was observed, resulting in a reduction of the total score of 8.1 and 4.2 points in the verum and placebo group, respectively, at the end of the study (Table 4).

In addition to the evaluation of the individual symptoms by medical investigators, the documentation in the patient diaries allowed an exact record of the dynamics and the recognition of an early complaints-free condition (Fig. 1). Up to day 7 of the study the evaluation of the five cold symptoms was almost completely documented twice daily in the diary by ninety-five patients $(96.8 \%)$. Highly significant differences $(P<0.01)$ between the groups in favour of the verum group were already shown after 3 to $4 \mathrm{~d}$, whereby the baseline situation for each cold symptom was not different in both groups. The sole exception was the highly significant improvement $(P<0.01)$ of the general feeling of sickness only on the fifth day. In summary, the improvement of cold symptoms was confirmed by the patients' self-rating.

Besides the documentation of the severity of cold symptoms, the medical investigators evaluated the existence of local findings. At the first examination all patients showed reddening of the Waldeyer's ring and/or the tonsils. By the end of the study a significant $(P<0.05)$ difference between the verum and the placebo groups was documented. While sixteen patients $(32.7 \%)$ receiving the verum drink were complaint free, only six patients $(12.8 \%)$ in the placebo group revealed no more reddening. Even a highly significant improvement $(P<0.01)$ could be observed concerning granulation and myxorrhoea on the back of the throat. Granulation on the back of the throat was only absent in six patients, three in each group, at the beginning of the study. Finally, forty $(81.6 \%)$ and twenty $(42.6 \%)$ patients in the verum and placebo group, respectively, exhibited no granulation. Comparable with the amelioration of granulation, forty-one patients of the verum group $(83.7 \%)$ and twenty-three patients of the placebo group (48.9\%) had no myxorrhoea on the back of the throat. At the beginning of the study, all participants revealed this finding with the exception of four patients in the verum group. Last, the percentage of patients without herpes labialis was significantly higher $(P<0.05)$ in the verum group (93\%) compared with in the placebo group $(79 \%)$.

\section{Relief of complaints at the end of the study (secondary efficacy criterion)}

The number of complaints-free individuals, determined by the physicians as well as by the patients themselves, was defined as the secondary efficacy criterion. At the third examination nineteen out of forty-nine patients $(38.8 \%)$ of the verum group and only four patients out of forty-seven $(8.3 \%)$ in the placebo group were without complaints $\left(P<0.001 ; \chi^{2}\right.$ test $)$ (Table 5). This significant difference was even more obvious in the case of the patients' evaluation. Hence, $41.9 \%$ of the patients taking verum reported to be complaint free contrary to $5.0 \%$ of patients of the placebo group on the evening of study day 7. Again, the superiority of the verum compared with the placebo could be proven (verum group $41.9 \% v$. placebo group 5.0\%; $P<0.001 ; \chi^{2}$ test).

Table 4. Decrease of total score of cold symptoms during the course of the study (primary efficacy criterion)

(Mean differences and standard deviations)

\begin{tabular}{|c|c|c|c|c|c|c|c|c|c|c|c|c|}
\hline \multirow[b]{2}{*}{ Time period } & \multicolumn{4}{|c|}{ Total collective } & \multicolumn{4}{|c|}{ Verum group } & \multicolumn{4}{|c|}{ Placebo group } \\
\hline & $n$ & Mean difference & SD & $P$ & $n$ & Mean difference & $\mathrm{SD}$ & $P$ & $n$ & Mean difference & $\mathrm{SD}$ & $P$ \\
\hline Examination 1 to 2 & 97 & $2 \cdot 4$ & $2 \cdot 4$ & 0.001 & 49 & 3.6 & $2 \cdot 3$ & 0.001 & 48 & 1.3 & 1.9 & 0.058 \\
\hline Examination 2 to 3 & 97 & $6 \cdot 2$ & $4 \cdot 2$ & 0.001 & 49 & 8.1 & 4.4 & 0.001 & 48 & 4.2 & $2 \cdot 7$ & 0.001 \\
\hline
\end{tabular}



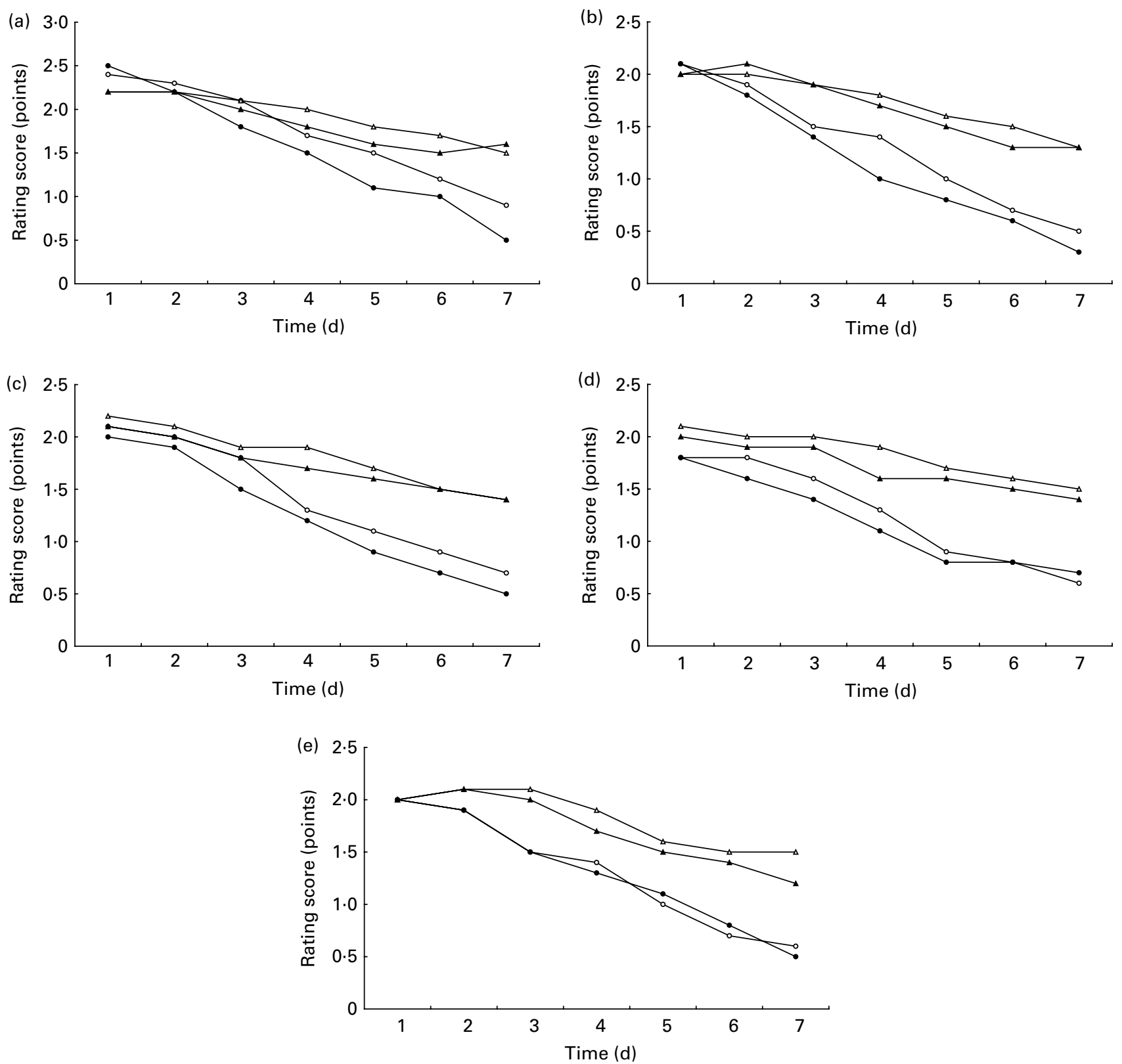

Fig. 1. Evaluation of the five cold symptoms comparing verum ( $\bigcirc$ morning, $\bullet$ evening) and placebo ( $\triangle$ morning, $\Delta$ evening) according to the patient diary in points. (a) General feeling of sickness, (b) headache and/or joint paints, (c) throat complaints, (d) hoarseness and/or cough, (e) nasal congestion/sniffle.

\section{Concurrent variables}

Sleep disorders and disturbances of daily activities are frequently accompanying symptoms of colds. In the present study more than 60 and $70 \%$ of the patients, respectively, suffered from these ailments. Starting on the third day of the study, a highly significant improvement $(P<0.01)$ in sleep disorders was recorded in the verum group, resulting in a reduction from 64.6 to $8.9 \%$ of affected patients on study day 7 (Fig. 2). Similarly, a highly significant improvement $(P<0.01)$ regarding disturbance of daily activities was observed on the fourth study day. On day 7 , a decrease from 87.5 to $9.1 \%$ of affected patients was documented in the verum group. On the contrary, 18.2 and $60.5 \%$ of patients treated with placebo still suffered from sleep disorders and disturbances of daily activities, respectively. Furthermore, the difference in average daily tissue use between both groups was highly significant $(P<0.01)$ on the seventh day. According to the patient diaries the utilisation in the verum group was only half of that in the placebo group.

No significant difference was noted between both groups with reference to additional cold therapy. The application of additional cold therapy was reduced from $39.6 \%$ at the beginning to $18.2 \%$ by the seventh day in the verum group and from 34.1 to $28.6 \%$ in the placebo group.

\section{Global efficacy rating by the physician and patient}

The physician rated the efficacy of the verum drink to treat symptoms of the common cold as 'good' or 'very good' in 42.9 and $34.7 \%$, respectively (Table 6). Similarly, $42.9 \%$ of 
Table 5. Relief of complaints at the end of the study (secondary efficacy criterion) (\%)

\begin{tabular}{|c|c|c|c|c|c|c|c|c|c|c|}
\hline & \multicolumn{10}{|c|}{ Sum score range } \\
\hline & 0 Points* & $P \dagger$ & 1-3 Points & $P \dagger$ & 4-6 Points & $P \dagger$ & 7-9 Points & $P \dagger$ & $>9$ Points & $P \dagger$ \\
\hline \multicolumn{11}{|c|}{ Clinical investigatorł } \\
\hline Verum $(n 49)$ & $38 \cdot 8$ & $<0.001$ & $36 \cdot 7$ & 0.084 & $14 \cdot 3$ & 0.554 & $8 \cdot 2$ & 0.045 & $2 \cdot 0$ & $<0.001$ \\
\hline Placebo ( $n$ 48) & $8 \cdot 3$ & & $20 \cdot 8$ & & $18 \cdot 8$ & & $22 \cdot 9$ & & $29 \cdot 2$ & \\
\hline \multicolumn{11}{|l|}{ Patient§ } \\
\hline Verum ( $n$ 43) & 41.9 & $<0.001$ & $32 \cdot 6$ & 0.062 & $18 \cdot 6$ & 0.872 & $7 \cdot 0$ & 0.013 & 0.0 & $<0.001$ \\
\hline Placebo (n 40) & $5 \cdot 0$ & & $15 \cdot 0$ & & $20 \cdot 0$ & & $27 \cdot 5$ & & 32.5 & \\
\hline
\end{tabular}

* 0 Points means free of complaints.

† Verum group $v$. placebo group ( $\chi^{2}$ test).

$\ddagger$ According to the evaluation on the third examination.

$\S$ According to the patient diary on the evening of day 7 .

the patients assessed the effectiveness as 'very good' and $30.6 \%$ as 'good'. In contrast to the verum treatment, the efficacy in the placebo group was considered as 'moderate' $(42.6 \%)$ and 'bad' $(31.9 \%)$ by the investigators. Even $40.4 \%$ of patients evaluated the effectiveness as 'bad'. Thus, both investigator and patient ratings showed the superiority of the verum treatment in comparison with placebo.

\section{Discussion}

The present study clearly indicates that patients suffering from common cold symptoms benefit from the consumption of the investigated polyphenol-rich and shitake extractcontaining beverage. Hence, patients receiving the verum
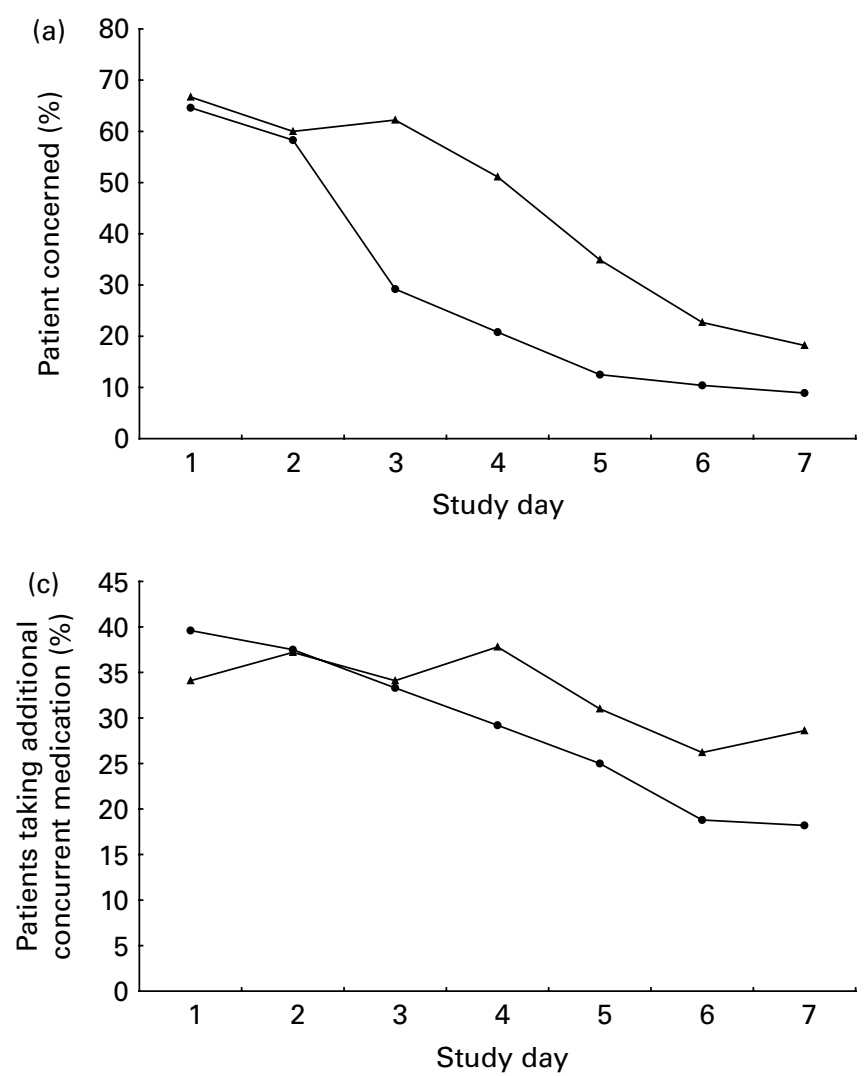

Fig. 2. Change of the concurrent variables comparing verum $(\bullet)$ and placebo medication' (c) and 'number of tissues used' (d) during the study. drink experienced both a faster decline of symptoms and were complaint free sooner compared with those taking the placebo. Since evaluation of the improvement was established both by patients and clinical investigators, not only selfreported values, which are often considered as 'soft' criteria, but also several local findings of a physical examination were significantly improved. At the end of the study, a reduction of the total score of the five cold symptoms from $10 \cdot 2$ (SD 3.1) to $2 \cdot 1$ (SD 2.7) points in the verum group and from 10.5 (SD 3.0) to only 6.3 (SD 3.9) points in the placebo group was observed. Due to the fact that the investigated beverage did not improve some specific symptoms but rather improved virtually all symptoms and constraints, there is no reason to assume that it provided a specific
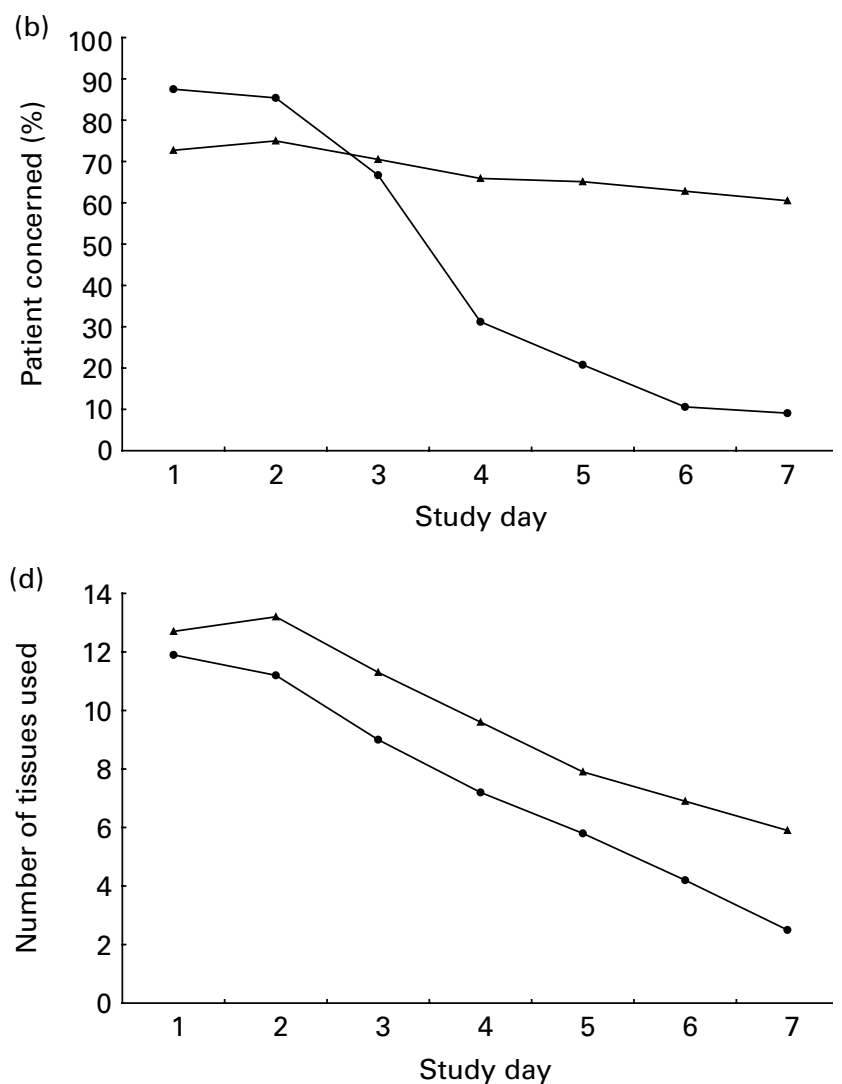
Table 6. Global efficacy rating (\%) by the physician and patient

\begin{tabular}{lccccc}
\hline & \multicolumn{2}{c}{ Verum } & & \multicolumn{2}{c}{ Placebo } \\
\cline { 2 - 3 } \cline { 5 - 6 } & $\begin{array}{c}\text { Clinical } \\
\text { investigator }\end{array}$ & Patient & & $\begin{array}{c}\text { Clinical } \\
\text { investigator }\end{array}$ & Patient \\
\hline Very good & 34.7 & 30.6 & & 8.5 & 8.5 \\
Good & 42.6 & 42.9 & & 17.0 & 17.0 \\
Moderate & 22.4 & 22.4 & & 42.6 & 34.0 \\
Bad & 0.0 & 4.1 & & 31.9 & 40.4 \\
\hline
\end{tabular}

therapeutic option. Therefore, it is much more likely that the polyphenol-rich drink stimulated and supported general defence and immunity. This assumption is further supported by the observation that subjects in the verum group showed a reduced susceptibility for herpes labialis. The percentage of subjects without herpes labialis $(93.9 \%)$ in the verum group was significantly higher compared with that in the placebo group $(79.2 \%)$ by the end of the study. Since herpes labialis symptoms reflect reactivation of existing viruses due to impaired immune defence it can be concluded that subjects in the verum group were less susceptible due to improved immune function.

This immune-modulating efficacy could be ascribed to the bioactive constituents of the investigated drink, particularly polyphenols from green tea and grapes. Thus, a positive influence of green tea catechins and L-theanin on the incidence and the duration of cold and flu symptoms were observed in a recent randomised, double-blind, placebo-controlled study involving 124 healthy participants. The results showed that treatment with green tea capsules reduced by about one-third the number of subjects with symptoms, and decreased the number of days that test individuals had symptoms as well as the number of patients requiring medication. Similarly, a significant improvement of cold symptoms in the verum group was already observed at the second examination (Table 4). Apart from these in vivo findings, the daily supplementation enhanced $\gamma \delta \mathrm{T}$ cell function $e x$ vivo, i.e. increased cell proliferation and IFN- $\gamma$ production, in response to antigen challenge. Taking into account that this subset of $\mathrm{T}$ cells plays a role as a first line of defence against infection, these data may explain the health benefits of green tea ${ }^{(41)}$. Moreover, from several non-human studies it can be concluded that green tea compounds modulate the body's defence by inducing anti-inflammatory responses as well as by exerting antiviral and antibacterial effects ${ }^{(13,16)}$.

In contrast to these findings for green tea, human intervention trials reporting a direct immune-modulatory effect of grape polyphenols are still lacking. Until now, several studies have proven the ability of grape polyphenols to alter antioxidative capacity in vivo ${ }^{(22,24)}$. For example, in a study including haemodialysis patients as well as healthy subjects, daily supplementation with $100 \mathrm{ml}$ red grape juice, corresponding to $640 \mathrm{mg}$ total phenols (GAE), for 2 weeks significantly increased the antioxidant capacity of plasma measured as Trolox-equivalent antioxidative capacity (TEAC) in both groups $^{(25)}$. Since this polyphenol intake was comparable with the amount of grape-derived polyphenols provided by the $500 \mathrm{ml}$ verum, similar results regarding antioxidative protection could be expected. Furthermore, on the basis of recent findings demonstrating a protective effect of grape seed-derived proanthocyanidin on oxidative DNA damage in rat leucocytes, an indirect way of supporting immune functions as an antioxidant could be assumed ${ }^{(42)}$. Furthermore, various studies have demonstrated the health-promoting potential of anthocyanins including anti-inflammatory activity $^{(43)}$. Cyanidin 3-O-glucoside, also commonly found in grapes ${ }^{(44)}$, inhibited cyclo-oxygenase enzymes COX-1 and COX-2 comparable with non-steroidal anti-inflammatory and analgesic drugs such as aspirin and ibuprofen, explaining the pronounced effect on headache and joint pains seen in the verum group (Fig. 1(b)) ${ }^{(45,46)}$.

Regarding shiitake mushrooms and lentinan, clinical data investigating their immune-modulating activity after oral application are still lacking. So far, lentinan has been used intravenously, intraperitoneally or intramuscularly as an adjuvant in the treatment of diseases involving depressed immune function $^{(32)}$. Only two studies examining the oral application of yeast-derived $\beta$-glucans are available. In a prospective clinical trial, the in vivo effects on peripheral blood monocytes and their expression of activation markers in twenty-three patients with advanced breast cancer were assessed. After 2 weeks of daily $20 \mathrm{mg} \mathrm{1-3,1-6} \beta$-glucan application, the mean monocyte count as well as the expression of markers identifying activated monocytes were significantly increased ${ }^{(47)}$. Further, the immunomodulating activity of a highly purified, soluble yeast $\beta$-glucan was studied in eighteen healthy participants assigned to three groups receiving 100, 200 or $400 \mathrm{mg}$ $\beta$-glucan per $d$, respectively. The dosage format was a mouthwash, which was consequently swallowed. At the end of the study, a significant increase of $\operatorname{IgA}$ in the saliva of the group receiving the highest dose of $\beta$-glucan solution was determined. Since $\operatorname{IgA}$ is released into the mucus of the nose, mouth, throat, lung or intestine and serves as a first barrier towards pathogens, the authors stated that any increase is appealing with regard to an oral colonisation by microorgansims such as Candida albicans ${ }^{(48)}$. Both studies have in common that higher concentrations of $\beta$-glucan compared with the amount of shiitake extract used in the present study were applied. Nevertheless, a higher concentration than $0.05 \mathrm{~g} / \mathrm{l}$ was not recommendable due to sensory acceptance, and hence, an immunomodulative activity appeared improbable. However, for an unambiguous clarification of whether shiitake extract in the present concentration exhibit any immune-supportive activity or not, further studies are required.

Besides extracts from green tea, grape skin and seeds, as well as shiitake, the verum drink contained vitamin $\mathrm{C}$, whose immunomodulatory effects, particularly by means of reduced susceptibility to infectious diseases and/or its efficacy on symptoms and duration of common colds, has been studied extensively ${ }^{(49,50)}$. However, in general, the results give no evidence that vitamin $\mathrm{C}$ supplementation is useful in common cold therapy, whereas it may have some effects to prevent infection in specific groups of individuals, such as subjects with low dietary vitamin $\mathrm{C}$ intake or exposed to physical or physiological stress ${ }^{(51)}$. Since the study population can be characterised as having impaired immune defence, similar to subjects eating an unbalanced diet or subjects exposed to physical stress, a pharmacological impact might be possible, even if the applied concentration of $61.4 \mathrm{mg}$ vitamin $\mathrm{C} / 500 \mathrm{ml}$ was less compared with the dosages normally used 
in human studies. Last, only by means of a further study including a placebo containing vitamin $\mathrm{C}$ could a possible effect be excluded. In fact, the addition of vitamin $\mathrm{C}$ was present due to some technological reasons, to protect the polyphenols against oxidation, rather than for a therapeutic effect. As can be seen from Table 2, a reduction from initially $300 \mathrm{mg}$ ascorbic acid/l to $122.7 \mathrm{mg} / \mathrm{l}$ occurred as consequence of oxidative processes during production. Further degradation of vitamin $\mathrm{C}$ as well as a change in polyphenol content during storage could be excluded through stability test over a time period of 9 months (data not shown).

In conclusion, the polyphenol-rich beverage exhibited a clear, positive impact on symptoms associated with the common cold through immunomodulative efficacy. Due to the exclusion criteria, the ailment might be considered as rather mild and the physiological functions of these patients had not been modulated by medication or severe organ or system disease. In fact, the study population can be characterised as having impaired immune defence, similar to subjects eating an unbalanced diet or exposed to physical stress, and thus, being sensitive for nutritional benefits. Therefore, the study population can be further considered as an appropriate target to demonstrate the clinical benefits of an immune stimulation and there is no reason to assume that the observed improvement cannot be extrapolated to the general population. Moreover, these benefits may be most obvious during periods of stress or illness. Hence, the results of the present study suggest that consumption of the investigated polyphenol-rich beverage strengthens the body's defence and improves immune function in the general population, which may translate into clinically measurable effects in individuals having impaired immune defence due to physical stress or already existing infections.

\section{Acknowledgements}

The authors thank Mrs Natalie Kernich for technical assistance.

The present study was funded by Rudolf Wild GmbH \& Co. $\mathrm{KG}$ and carried out by analyze \& realize ag.

Authors' contributions are acknowledged as follows: K. S. was the main writer of the manuscript; M. S. had the original idea, developed the test beverage and managed the study for Rudolf Wild GmbH \& Co. KG; A. de W. proofread the manuscript; H.-J. G. and J. G. conducted the experimental work and performed the statistical analyses. All authors saw the final draft.

None of the authors has any conflicts of interest in the writing and publication of the present study.

\section{References}

1. Heikkinen T \& Järvin A (2003) The common cold. Lancet 361, 51-59.

2. Eccles R (2005) Understanding the symptoms of the common cold and influenza. Lancet Infect Dis 5, 718-725.

3. Craig WJ (1999) Health-promoting properties of common herbs. Am J Clin Nutr 70, 491S-499S.

4. Roxas M \& Jurenka J (2007) Colds and influenza: a review of diagnosis and conventional, botanical, and nutritional consideration. Altern Med Rev 12, 25-47.
5. Zakay-Rones Z, Varsano N, Zlotnik M, et al. (1995) Inhibition of several strains of influenza virus in vitro and reduction of symptoms by an elderberry extract (Sambucus nigra L.) during an outbreak of influenza B Panama. J Altern Complement Med 1, 361-369.

6. Zakay-Rones Z, Thom E, Wollan T, et al. (2004) Randomized study of the efficacy and safety of oral elderberry extract in the treatment of influenza A and B virus infections. J Int Med Res 32, 132-140.

7. Wichtl M (editor) (1989) Elderberry, elder flowers. In Teedrogen - Ein Handbuch für die Praxis auf wissenschaftlicher Grundlage (Tea Drugs - A Handbook for Practice on a Scientific Basis), 2nd ed., pp. 237-241. Stuttgart: Wissenschaftliche Verlagsgesellschaft $\mathrm{mbH}$.

8. Lindenmuth GF \& Lindenmuth EB (2000) The efficacy of Echinacea compound herbal tea preparation on the serverity and duration of upper respiratory and flu symptoms: a randomized, double-blind placebo-controlled study. J Alter Complement Med 4, 327-334.

9. ESCOP (2003) 'Echinaceae pallidae radix', 'Echinaceae purpureae radix' and 'Echinaceae purpureae herba'. In Monographs on the Medicinal Uses of Plant Drugs, 2nd ed., pp. 126-141, European Scientific Cooperative on Phytotherapy: Thieme Stuttgart.

10. Kaul TN, Middleton E Jr \& Ogra PL (1985) Antiviral effect of flavonoids on human viruses. J Med Virol 15, 71-79.

11. Franz G (1995) Natural products with immunomodulating and antineoplastic activity. Pharm Pharmacol Lett 5, 154-158.

12. Middleton E Jr (1998) Effect of plant flavonoids on immune and inflammatory cell function. Adv Exp Med Biol 439, 175-182.

13. Song JM \& Seong BL (2007) Tea catechins as a potential alternative anti-infectious agent. Expert Rev Anti Infect Ther 5, 497-506.

14. Hu ZQ, Toda M, Okuba S, et al. (1992) Mitogenic activity of $(-)$ epigallocatechin gallate on B-cells and investigation of its structure-function relationship. Int J Immunopharmacol 14, 1399-1407.

15. Kamath AB, Wang L, Das H, et al. (2003) Antigens in teabeverage prime human $\mathrm{V} \gamma 2 \mathrm{~V} \delta 2 \mathrm{~T}$ cells in vitro and in vivo for memory and nonmemory antibacterial cytokine responses. Immunology 13, 6009-6014.

16. Clarke JO \& Mullin GE (2008) A review of complementary and alternative approaches to immunomodulation. Nutr Clin Pract 23, 49-62.

17. Nakayama M, Suzuki K, Toda M, et al. (1993) Inhibition of the infectivity of influenza virus by tea polyphenols. Antiviral Res 21, 289-299.

18. Imanishi N, Tuji Y, Katada Y, et al. (2002) Additional inhibitory effect of tea extract on the growth of influenza A and B viruses in MDCK cells. Microbiol Immunol 46, 491-494.

19. Ikigai H, Nakae T, Hara Y, et al. (1993) Bactericidal catechins damage the lipid bilayer. Biochim Biophys Acta 1147, 132-136.

20. Yamada H, Takuma N, Daimon T, et al. (2006) Gargling with tea catechin extracts for the prevention of influenza infection in elderly nursing home residents: a prospective clinical study. J Altern Complement Med 12, 669-672.

21. Bub A, Watzl B, Blockhaus M, et al. (2003) Fruit juice consumption modulates antioxidative status, immune status and DNA damage. J Nutr Biochem 14, 90-98.

22. Whitehead TP, Robinson D, Allaway S, et al. (1995) Effect of red wine ingestion on the antioxidant capacity of serum. Clin Chem 41, 32-35.

23. Nutall SL, Kendall MJ, Bombardelli E, et al. (1998) An evaluation of the antioxidant activity of a standardized grape seed ectract, Leucoselect ${ }^{\circledR}$. J Clin Pharm Ther 23, 385-389.

24. O'Byrne DJ, Devaraj S, Grundy SM, et al. (2002) Comparison of the antioxidant effects of Concord grape juice flavonoids and 
$\alpha$-tocopherol on markers of oxidative stress in healthy adults. Am J Clin Nutr 76, 1367-1374.

25. Castilla P, Echarri R, Dávalos A, et al. (2006) Concentrated red grape juice exerts antioxidant, hypolipidemic, and antiinflammatory effects in both hemodialysis patients and healthy subjects. Am J Clin Nut 84, 252-262.

26. Netzel M, Netzel G, Maier T, et al. (2008) Polyphenole aus Trauben - Erste Ergebnisse aus Metabolisierungsstudien mit Traubentresterextrakten und Probanden (Polyphenols from grapes - early results from metabolism studies with grape pomace extracts and volunteers). Fluess Obst 5, 240-246.

27. Zhang XY, Li WG, Wu YJ, et al. (2005) Amelioration of doxorubicin-induced myocardial oxidative stress and immunosuppression by grape seed proanthocyanidins in tumour-bearing mice. J Pharm Pharmacol 57, 1043-1051.

28. Nair N, Mahajan S, Chawda R, et al. (2002) Grape seed extract activates Th1 cells in vitro. Clin Diagn Lab Immunol 9. $470-476$

29. Konowalchuk J \& Speirs JI (1976) Virus inactivation by grapes and wines. Appl Environ Micorbiol 32, 757-763.

30. Nair MP, Kandaswami C, Mahajan S, et al. (2002) Grape seed extract proanthocyanidins downregulate HIV-1 entry coreceptors, CCR2b, CCR3 and CCR5 gene expression by normal peripheral blood mononuclear cells. Biol Res 35, 421-431.

31. Jayaprakasha GK, Selvi T \& Sakaria KK (2003) Antibacterial and antioxidant activities of grape (Vitis vinifera) seed extracts. Food Res Int 36, 117-122.

32. Wasser SP (2005) Shiitake (Lentinus edodes). In Encyclopedia of Dietary Supplements, pp. 653-664 [P Coates, MR Blackman, G Cragg, M Levine, J Moss and J White, editors]. New York: Marcel Dekker.

33. Chen J \& Seviour R (2007) Medicinal importance of fungal $\beta$ - $(1 \rightarrow 3),(1 \rightarrow 6)$-glucans. Mycol Res 111, 635-652.

34. Cummings JH, Antoine JM, Azpiroz F, et al. (2004) PASSCLAIM - Gut health and immunity. Eur J Nutr 43, Suppl. 2, II/118-II/173.

35. Albers R, Antoine JM, Bourdet-Sicar R, et al. (2005) Markers to measure immunomodulation in human nutrition intervention studies. Br J Nutr 94, 452-481.

36. Miller NJ, Rice-Evans C, Davies MJ, et al. (1993) A novel method for measuring antioxidant capacity and its application to monitoring the antioxidant status in premature neonates. Clin Sci 84, 407-412.

37. Benzie IF \& Strain JJ (1996) The ferric reducing ability of plasma (FRAP) as a measure of 'antioxidant power': the FRAP assay. Anal Biochem 239, 70-76.

38. Popov I \& Lewin G (1999) Antioxidative homeostasis: characterization by means of chemiluminescent technique. Methods Enzymol 300, 437-456.
39. Singleton VL \& Rossi JA (1965) Colorimetry of total phenolics with phosphomolybdic-phosphotungstic acid reagents. Am J Enol Vitic 16, 144-158.

40. Ross MA (1994) Determination of ascorbic acid and uric acid in plasma by high-performance liquid chromatography. J Chromatogr B 657, 197-200.

41. Rowe CA, Nantz MP, Bukowski JF, et al. (2007) Specific formulation of Camellia sinensis prevents cold and flu symptoms and enhances $\gamma \delta \mathrm{T}$ cell function: a randomized, doubleblind, placebo-controlled study. J Am Coll Nutr 26, 445-452.

42. Morin B, Narbonne JF, Ribera D, et al. (2008) Effect of dietary fat-soluble vitamins $\mathrm{A}$ and $\mathrm{E}$ and proanthocyanidin-rich extract from grape seeds on oxidative DNA damage in rats. Food Chem Toxicol 46, 787-796.

43. Ghosh D (2005) Anthocyanins and anthocyanin-rich extracts in biology and medicine: biochemical, cellular, and medicinal properties. Curr Top Nutraceutical Res 3, 113-124.

44. Kammerer D, Claus A, Carle R, et al. (2004) Polyphenol screening of pomace from red and white grape varieties (Vitis vinifera L.) by HPLC-DAD-MS/MS. J Agric Food Chem 52, 4360-4367.

45. Reddy MK, Alexander-Lindo BL \& Nair MG (2005) Relative inhibition of lipid peroxidation, cyclooxygenase enzymes, and human tumor cell proliferation by natural food colors. J Agric Food Chem 53, 9268-9273.

46. Adhikari DP, Francis JA, Schutzki RE, et al. (2005) Quantification and characterisation of cyclooxygenase and lipid peroxidation inhibitory anthocyanins in fruits of Amelanchier. Phytochem Anal 16, 175-180.

47. Demir G, Klein HO, Mandel-Molinas N, et al. (2007) $\beta$ Glucan induces proliferation and activation of monocytes in peripheral blood of patients with advanced breast cancer. Int Immunopharmacol 7, 113-116.

48. Lehne G, Haneberg B, Gaustad P, et al. (2006) Oral administration of a new soluble branched $\beta-1,3$-D-glucan is well tolerated and can lead to increased salivary concentration of immunoglobulin A in healthy volunteers. Clin Exp Immunol 143, 65-69.

49. Hemila H (1997) Vitamin $C$ intake and susceptibility to the common cold. Br J Nutr 77, 59-72.

50. Moreira A, Kekkonen RA, Delgado L, et al. (2007) Nutritional modulation of exercise-induced immunodepression in athletes: a systematic review and meta-analysis. Eur J Clin Nutr 61, 443-460.

51. Hemilä H, Chalker E \& Douglas RM (2007) Vitamin C for preventing and treating the common cold. Cochrane Database of Systematic Reviews, issue 3, CD000980. http://www.mrw. interscience.wiley.com/cochrane/clsysrev/articles/CD000980/ frame.html 